\title{
Research and Modeling on Self-tuning Dynamic Control System of Flight Simulator Based on PID Neural Network
}

\author{
Daoyang Zhu' ${ }^{a}$, Da Fang, Yan Ma, Shaoli Duan \\ Wuhan Technical College of Communications, Wuhan, Hubei 43000, China \\ adyzhu_xg@aliyun.com
}

Keywords: flight simulation; PID control; BP neural network; self-tuning control

\begin{abstract}
The accuracy of PID control system is a little poor on Stewart platform, and parameters adjustment of PID controller cannot be adjusted in real time online, by combining the advantage of BP neural network, which is good self-learning, selfadaptation, and it can approach any nonlinear system with arbitrary precision. The closed loop error between directly measured output and desired value of system, and desired length is chosen to be the input of controller ,in order to achieve self-tuning real-time online of controller parameters for motion platform, improve the accuracy of control system at the same time. The dynamic simulation model of flight simulator based PID neural network is built and utilized to verify the reliability in Matlab/Simluink. The result shows that the PID neural network control algorithm can achieve adaptive parameter adjustment, and system error have dropped significantly after neural network training. It is proved that the PIDNN controller can achieve self-tuning real-time online of the parameter, and the system has good adaptability and robustness.
\end{abstract}

\section{Introduction}

At present, most of the motion platform of flight simulator have applied Stewart parallel motion mechanisms, because it has advantages of the better security, large load capacity, accurate position, no accumulation error and so on [1].Electro-hydraulic servo control system in Stewart platform has been found to best meet the performance requirements for many years [2][3], by regularly controlling the length of hydraulic cylinder in the Stewart platform, flight simulator can fully replicate the actual movement, thus the Stewart platform is usually known as the six-degree-offreedom (6-DOF) motion platform. The performance requirement for 6-DOF motion platform in terms of high accuracy, good adaptability and robustness have generated continuous challenges in the field of motion control system, because 6-DOF motion platform is a time-varying nonlinear process control multi-input/multi-output (MIMO) system[4][5]. In motion platform control applications, proportional-integral-derivative (PID) control is widely used as a control method because of its simple architecture, high stability and strong robustness [6]. However, 6-DOF motion platform has demonstrated significant internal interaction, because each every hydraulic cylinder need be driven by individual actuator arrangement. Engineers choose the method that trial and error techniques are inadequate to derive a good compromise between controller performance and robustness [7][8]. In addition,PID controller parameters need to be set offline and the adjustment 
process is complicated,and the calculation is large.These problems have been plaguing engineers, resulting in high engineering cost, low efficiency, and poor control accuracy,in order to overcome the problems, PID controllers that mixed neural network control [9][10], adaptive control [11], fuzzy control [12] were presented to researchers. With advantages like the abilities of adaptive learning, fault tolerance and generalization, artificial neural networks combines with PID control, which is one of the popular methods used for complexes nonlinear control system in design and application fields. Cong and Liang [13] studied the effect of learning rates based on PID-like neural network adaptive controller (PIDNNC) for multivariable SIMO systems with uncertainty and disturbance. The updating weights of PIDNN should take consideration of uncertain factors, such as modeling error and external disturbance.

In this paper, flight simulator dynamics control system model is built in Matlab/Simulink based on PIDNN. The method that PID control combines with BP neural network is utilized to deal with this problems,and two typical control algorithm are implemented and compared to verify the method.

\section{Flight Simulator Control System}

Firstly, the driver accepts the task and output the operating signal into flight simulator dynamics model.With the limited worksapce of motion platform, it can not completely repuduce aircraft's moving,so washout algorithm can be applied in the motion simulator [14 16],the instantaneous acceleration of aircraft is simulated to get the displacement in the high-pass acceleration channel, including surge, sway and heave. The principle of tilt coordination in the tilt coordination channel is used to simulate the continuous acceleration of aircarft. The angular displacement of motion platform is washed out by the tilt coordination channel and the high-pass angular velocity channel in the pitch, roll and yaw direction. Then displacement and angular displacement would obatin the hydraulic cylinder length of 6-DOF motion platform by kinematics inverse solution. Finally, PID controller achieve the closed loop control of the length change to control system steadily,the structure is showed in figure 1.

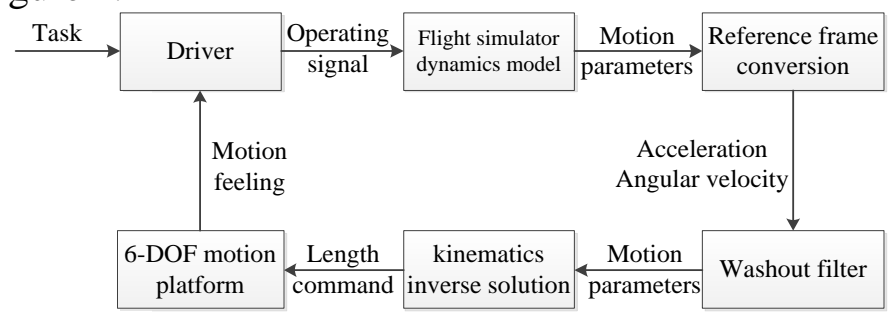

Figure 1 Flight simulator control system

\section{Self-tuning PID Neural Networks}

Since Rumelhart et al. proposed a multi-layer back propagation (BP) neural network, it has become the most widely used artificial neural network model because of its self-organization, selflearning, self-adaptation and the ability to approximate any nonlinear continuous function with arbitrary precision [17]. The BP neural network usually includes an input layer, an intermediate layer (hidden layer), and an output layer. Each layer has several neurons, and the layers are completely connected with each other. The upper and lower neurons of the same layer are not connected, and the hidden layer may be one or multilayer structure. There are a feedforward propagation and an error back propagation signal response between the layers. Because the PID controller parameters cannot be real time online adaptively adjusted in the flight simulator motion control system, so that the system control accuracy is low. This paper proposes and establishes a 
PID neural network controller to control the motion platform. The model is shown in Figure 2.

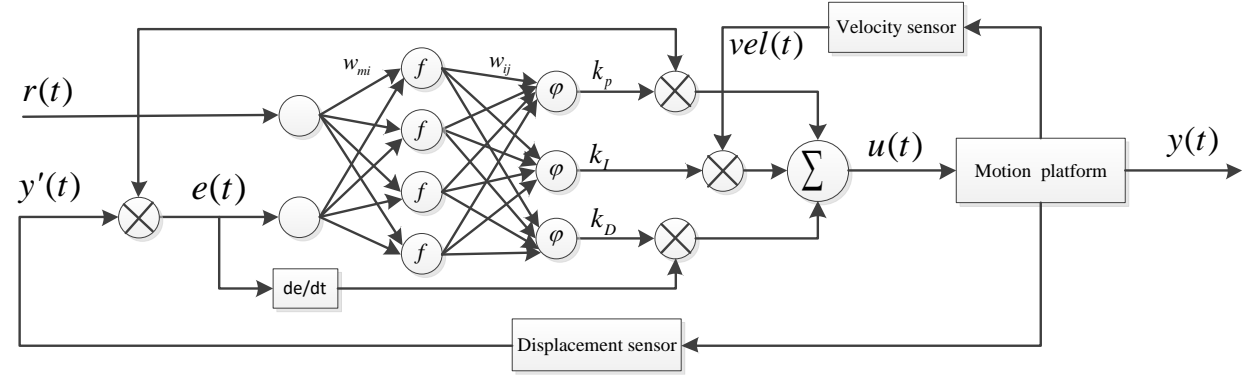

Figure 2 Structure of PID neural network

According to the strcture described above,the input of nodes in input layer $\boldsymbol{r}_{\boldsymbol{i}}(t)$ and $\boldsymbol{e}_{\boldsymbol{i}}(t),(i=1,2 \ldots 6)$, where vector $\boldsymbol{r}$ represents the desired displacement of the six hydraulic cylinders stroke length, the output error of closed loop system is described by vector $\boldsymbol{e}$. The weight value is $w_{\mathrm{mi}}$ and $w_{\mathrm{ij}}$ between input layer, hidden layer and output layer,respectively. Let the activation function of the hidden layer and the output layer be an S-function $f(x)=\varphi(x)=1 /\left(1+e^{-x}\right)$,so the output of the output layer are as follows:

$$
\left[k_{P}, k_{I}, k_{D}\right]=\sum_{j=1}^{3}\left(\varphi_{j}\left(\sum_{i=1}^{4} w_{i j} f_{i}\left(\sum_{m=1}^{2} w_{m i} x_{m}\right)\right)\right)
$$

Where, vector $k_{\mathrm{p}}, k_{\mathrm{I}}$ and $k_{\mathrm{D}}$ are the input parameters of the PID controller, and the final output of PID controller is

$$
u(t)=k_{p} e(t)+k_{I} \operatorname{vel}(t)+k_{D}(d e / d t)
$$

The system performance cost function is as follows, where $i$ represents the amount of six hydraulic cylinders:

$$
E(t)=\frac{1}{2} \sum_{n=1}^{6} e_{n}(t)^{2}=\frac{1}{2} \sum_{n=1}^{6}\left(r_{n}(t)-y_{n}^{\prime}(t)\right)^{2}
$$

Without loss of generality,the rules of upating weigths are obtaibed by means of minimizing the cost function $E(t)$.Here,the method of gradient descent are adopted to get updating rules. The weight values are updated from the output layer $J$ to the hidden layer $I$ :

$$
\left.\frac{\partial E(t)}{\partial w_{i j}(t)}=-\left(\sum_{n=1}^{6} e_{n}(t) \cdot\left(v_{j}^{J}(t)\right)^{\prime}\right)\right) \cdot v_{i}^{I}(t)
$$

The final rule of updating weigths from the hidden layer $I$ to the input layer $M$, as shown in the following formula 5:

$$
\left.\frac{\partial E(t)}{\partial w_{m i}(t)}=-\left(\sum_{n=1}^{6} e_{n}(t) \cdot\left(v_{j}^{J}\right)^{\prime}\right)\right) \cdot f_{i}^{\prime}\left(\sum_{m=1}^{2} w_{m i}(t) x_{m}(t)\right) \cdot w_{i j}(t) \cdot x_{m}(t) \quad(i=1,2,3,4 ; \quad j=1,2,3)
$$

Where, $v_{i}^{I}=f_{i}\left(\sum_{m=1}^{2} w_{m i} x_{m}\right)$ and $v_{j}^{J}=\varphi_{j}\left(\sum_{i=1}^{4} w_{i j} v_{i}^{I}\right)$ represent the output of the hidden layer and output layer, respectively.so that (6) can commonly express the rule of updating weigths in the hidden layer and output layer.

$$
w(t+\Delta T)=\alpha w(t)+\Delta w(t)
$$

Where $\Delta w(t)=-\eta \cdot(\partial E / \partial w)$, in which $\alpha$ is the momentum factor and $\eta$ is the learning rate. 


\section{Simulation model and simulation results}

In [18],the paper builds the flight simulator control system model based on PID control,but the PID control can achieve the goal tediously.By comparing,the dynamic verification model of flight simulator is established in Matlab/Simulink in figure 3.The control system mainly splitted into four components:washout algorithm,kinematics inverse solution,PIDNN controller and motion platform. According to the classical washout algorithm, displacement and angular displacement are obtained to adjust the attitude of Stewart mtion platform.The desired length variation and the length of six hydraulic cylinders can be computed by the kinematic inverse solution, and they will be accepted as the input parameters of BPNN and PID controller.Then, the actual length variation and velocity of the stroke are measured by sensors in the cylinder of the motion platform. Parameters $K_{P}, K_{I}$ and $K_{D}$ of PID controller are real-time online adaptive adjusted by BP neural network, and the force computed by PIDNN controller drives the electro-hydraulic servo system of motion palatform.Finally,the PID-BPNN controller can continuously reduce the accumulated error during the process of motion simulation, to precisely achieve the purpose that the dynamic fidelity of flight simulator can be developed.

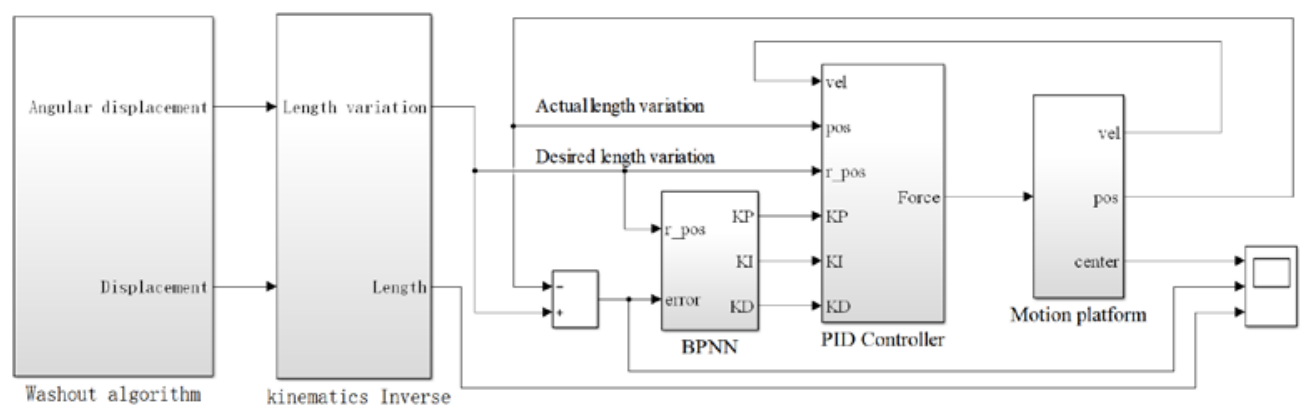

Figure 3 Flight simulator control system model

In order to ensure the accuracy of the flight simulation, acceleration of the surge direction is selected, the acceleration and angular velocity are zero in other directions. The signal of input simulation acceleration is shown in figure 4. The stroke length of the six hydraulic cylinders is calculated by the classical washout algorithm and the kinematics inverse solution of the motion platform. Figure 5 shows the desired stroke length variation. Comparatively, the BP neural network that gradient descent method is used to train the parameters of the PID controller implements the real-time online self-tuning of the PID parameters based BPNN control.

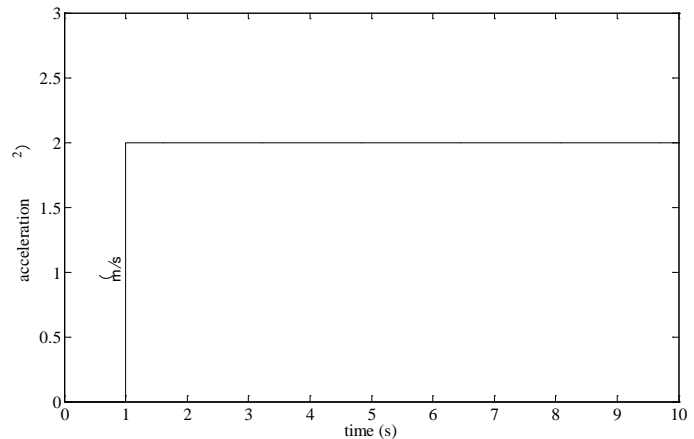

Figure 4 simulation acceleration

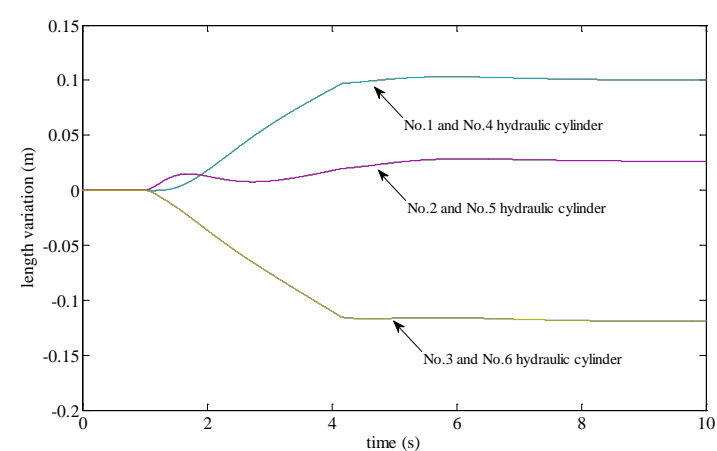

Figure 5 desired stroke length variation

In figure 6,dashed line represents PID controller system,solid line represnets PIDNN controller system,the length variation error of the PIDNN algorithm is significantly lower than that of the PID 
algorithm. Duing to the initial weight value selection of the BP neural network, all of the initial fluctuation of the length variation error is a little vibration at the beginning, but its margin can be quickly reduced by the training of BP neural network, and the fluctuation of later margin is no more than 10-7 after 0.1 seconds.it demonstrates that BPNN controller has superior adaptive performance and the accuracy of control system has significantly improvement.As can be seen form figure 7, by comparing the value of cost function E(t),PID algorithm sharply shocks in the early period and gradually stabilizes in the position of 10-5 after 3 second.The experiment verifies that PIDNN can effectively guaranteed system accuracy,and inherit the self-learning of BP neural network. The adaptivity and robustness of PIDNN control system can achieve better effectness with the flight simulator.
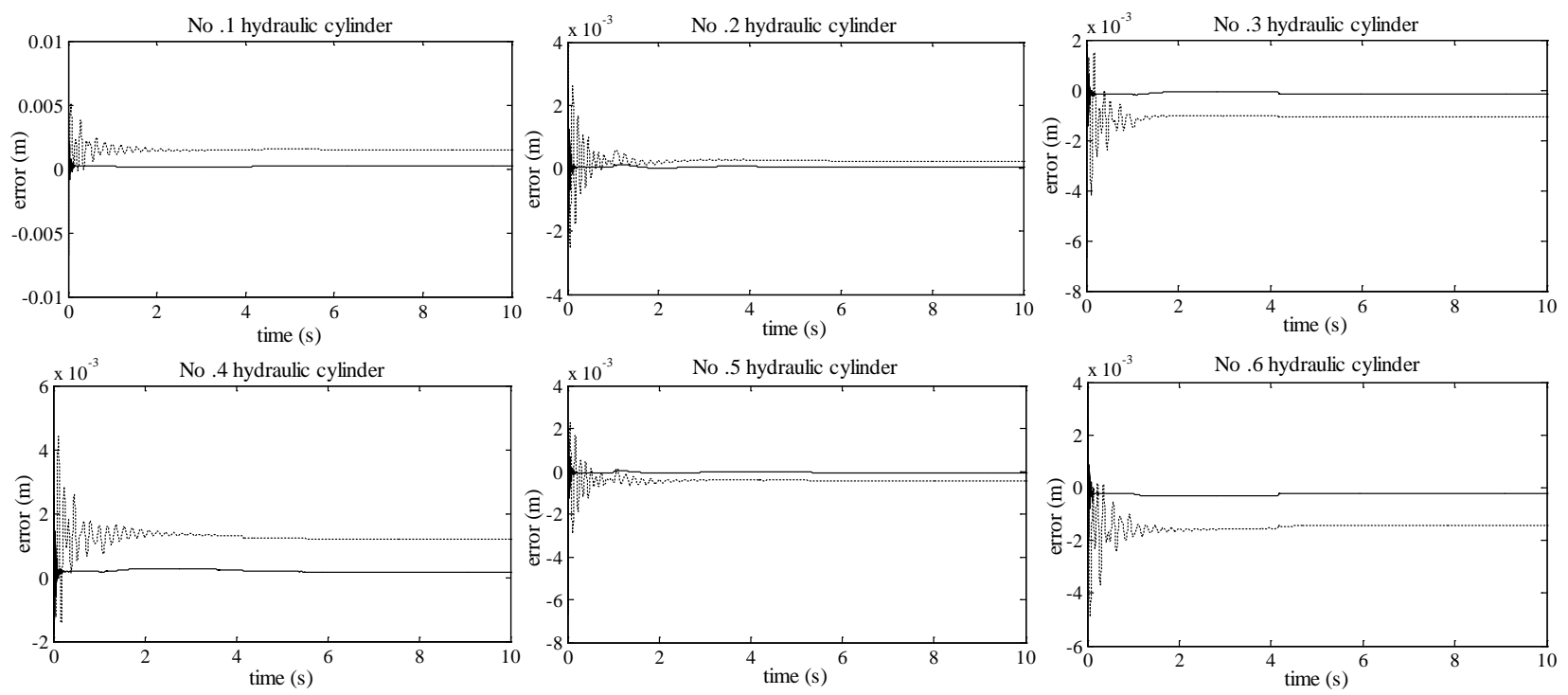

Figure 6 PID and PIDNN length variation error

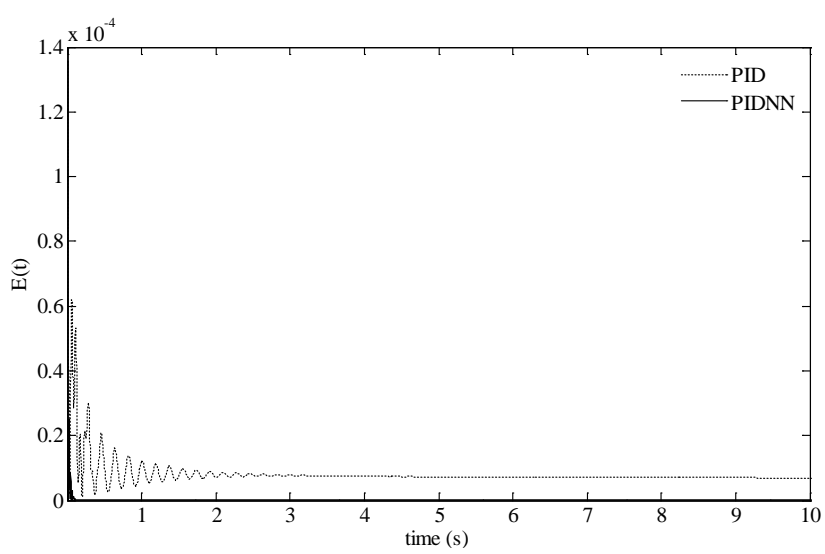

Figure 7 Performance value of the system

\section{Conclusion}

The self-tuning PIDNN algorithm that PID control combines with the advantages of BP neural network is proposed to solve the problems in this paper, which parameters of PID controller can't be adaptive adjusted in real time online, and the accuracy of PID control system is poor on Stewart platform. The paper builds the closed loop dynamic control system of flight simulator by PIDNN,the result is a proof that the method presented is not only effective for adaptive parameter 
adjustment and improves the control accuracy of system, but also effective for the process robustness.

\section{Acknowledgments}

This work was financially supported by Wuhan technical college of comunications fund (q2018001) and China institute of comunications education fund (1602-6) which also supports the project "Cultivation and practice of intelligent innovative talents based on 3D printing technology".

\section{References}

[1] Liu M J, Li C X, Li C N. Dynamics analysis of the Gough-Stewart platform manipulator [J]. Robotics \& Automation IEEE Transactions on, 2000, 16(1):94-98.

[2] Stewart D. A Platform with Six Degree of Freedom [J]. Proceedings of the Institute of Mechanical Engineering, 1965, Vol 180 Pt 1, No 15, p371-378.

[3] Haber R N. Flight simulation [J]. Scientific American, 1986, 255(1):96-103.

[4] Su Y X, Duan B Y, Zheng C H, et al. Disturbance-rejection high-precision motion control of a Stewart platform [J]. IEEE Transactions on Control Systems Technology, 2004, 12(3):364-374.

[5] Zhao D, Li S, Gao F. Fully Adaptive Feedforward Feedback Synchronized Tracking Control for Stewart Platform Systems [J]. International Journal of Control Automation \& Systems, 2008, 6(5):689-701.

[6] Ang K H, Chong G, Li Y. PID control system analysis, design, and technology [J]. IEEE Transactions on Control Systems Technology, 2005, 13(4):559-576.

[7] Lou J H, Tseng S P. Developing a real-time serial servo motion control system for electric stewart platform[C]// International Conference on Advanced Robotics and Intelligent Systems. IEEE, 2014:66-71.

[8] Davliakos I, Papadopoulos E. A model-based impedance control of a 6-dof electrohydraulic stewart platform[C]// Control Conference. IEEE, 2007:3507-3514.

[9] Zribi A, Chtourou M, Djemel M. A New PID Neural Network Controller Design for Nonlinear Processes [J]. Computer Science, 2015.

[10] Feng S, Xu M, Zhao D, et al. Research on RBF-PID Control for the 6-DOF Motion Base in Construction Telerobot System[C]// Computational Engineering in Systems Applications, IMACS Multiconference on. IEEE, 2006:19151920.

[11] Huang S N, Tan K K, Lee T H. A combined PID/adaptive controller for a class of nonlinear systems [J]. Automatica, 2001, 37(4):611-618.

[12] Zhu D Q, Xie W H, Sun L. Fuzzy Immune PID Control for 6-DOF Parallel Platform[J]. Applied Mechanics \& Materials, 2011, 128-129(25):890-893.

[13] Cong S, Liang Y. PID-Like Neural Network Nonlinear Adaptive Control for Uncertain Multivariable Motion Control Systems [J]. IEEE Transactions on Industrial Electronics, 2009, 56(10):3872-3879.

[14] Ariel, D. and Sivan, R., False Cue Reduction in Moving Flight Simulators, IEEE Transactions on Systems, Man and Cybernetics, SMC-14, No.4 (1984), pp.665-671

[15] Wu, W. and Cardullo, F.M., Is There an Optimum Cueing Algorithm, AIAA Modeling and Simulation Technologies Conference, New Orleans, LA, (1997),pp.23-29.

[16] Chen S H, Fu L C. An Optimal Washout Filter Design with Fuzzy Compensation for a Motion Platform [J]. IFAC Proceedings Volumes, 2011, 44(1):8433-8438.

[17] Kumar V, Gaur P, Mittal A P. ANN based self-tuned PID like adaptive controller design for high performance PMSM position control [J]. Expert Systems with Applications, 2014, 41(17):7995-8002.

[18] Daoyang zhu. Research on some key technologies of six-DOF motion hydraulic platform [D]. Civil Aviation University of China, 2017. 